\section{P90 Latino Father Self-Efficacy for Promoting Healthy Adolescent Behaviors was Associated with Frequency of Parenting Practices}

Azzairia Jackson, BS, University of Minnesota, Department of Food Science and Nutrition; Alejandro Reyes Peralta, $M P H$, University of Minnesota, Extension Center for Family Development; Ghaffar Ali Hurtado Choque, PhD, University of Maryland, School of Public Health; Marla Reicks, PhD, RDN, mreicks@umn.edu, University of Minnesota, 1334 Eckles Ave, St. Paul, MN, 55108

Background: Hispanic/Latino adolescents have a high prevalence of overweight/obesity and many are not meeting diet and physical activity (PA) recommendations. Latino father food and PA parenting practices can impact adolescent diet and PA behaviors. Paternal self-efficacy (PSE) for promoting healthy eating and PA may enhance frequency of positive food and PA parenting practices and healthy youth behaviors, yet little information is available about these relationships and change in PSE after intervention programs.

Objective: Evaluate relationships between PSE for promoting healthy behaviors, father food and PA parenting practices, and adolescent diet and PA, and interventionbased change in PSE.

Study Design, Setting, Participants: Baseline ( $n=190$ father/youth (10-14 years) dyads) and intervention data ( $\mathrm{n}=110$ dyads) were used from the communitybased, Padres Preparados, Jóvenes Saludables program, a randomized, controlled trial in Minneapolis/St. Paul (2017-2020). Eight-weekly education sessions focused on parenting skills and practices, nutrition and PA. Evaluation survey data were collected pre-post program.

Measurable Outcome/Analysis: PSE, parenting practices, and youth behaviors were measured with scales with acceptable psychometric properties and 24-hour dietary recalls. Regression analyses examined associations between PSE, parenting practices and youth behaviors. Interventionbased change in PSE were evaluated by $t$ tests.

Results: Mean youth age was 11.6 years, 49\% were boys. Adolescent screen time was negatively associated with PSE for promoting PA. Increased PSE was positively associated with increased modeling of fruit and vegetable intake and PA and with making fruit and vegetables and PA opportunities available. PSE for promoting PA increased from pre to post program for intervention compared to control group fathers.

Conclusion: Confidence in the ability to promote healthy youth lifestyle behaviors was associated with increased frequency of positive paternal modeling and availability practices. PSE for promoting PA improved from pre to post program. Educators could include strategies to promote PSE in future educational programs.

Funding: NIFA.
P91 Menu Quality and Food Substitution in Early Care and Education (ECE) Programs: The FRESH Study

Divya Patel, MS, divya-k-patel@ouhsc.edu, University of Oklahoma Health Sciences Center, 865 Research Pkwy, Oklahoma City, OK, 73104; Susan Sisson, PhD, CHES, RDN, University of Oklahoma Health Sciences Center; Kaysha

Sleet, BS, Oklahoma State University; Rachel Rickman, MS, Oklahoma State University; Charlotte Love, MPH, Oklahoma State University; Tori Taniguchi, MPH, Oklahoma State University; Valerie Blue Bird Jernigan, $\mathrm{DrPH}, \mathrm{MPH}$, Oklahoma State University

Background: Preschool children consume two thirds of their dietary requirements at Early Care and Education (ECE) programs. Programs in tribal communities face unique challenges like rural location and reduced food access. Evaluation of ECE menus is an efficient method to examine dietary quality of foods and find opportunity to enhance nutrition quality through training and interventions for programs including those serving Native American children.

Objective: Determine nutrient content, Child and Adult Care Food Program (CACFP) compliance, food substitution to assess menu quality in tribally affiliated ECE programs.

Study Design, Setting, Participants: Cross-sectional analysis was conducted as the baseline assessment in FRESH, a community-based participatory research study. Menus and recipes were collected from 9 sites and foods served to children were photographed.

Measurable Outcome/Analysis: Food Processor was used for nutrient analyses of fiber, saturated fat, and energy content. A quantitative index assessed the revised 2017 CACFP requirement, best practice, and combined compliance (possible score of zero to 100 percent for each). Food substitutions were classified as nutritionally equivalent, nutritionally superior (higher nutrient quality), or nutritionally inferior (poorer nutrient quality), and summed. Means, standard deviation, medians, and interquartile ranges were calculated for all variables.

Results: Mean fiber, saturated fat, and energy content of menus were $3.5 \mathrm{~g} \pm 0.5 \mathrm{~g}, 10.5 \mathrm{~g} \pm 3.4 \mathrm{~g}$, and $643.7 \pm 106.6$ kcals, respectively. Menus were $74.4 \%, 61.4 \%$, and $88.1 \%$ compliant with the total CACFP guidelines, best practices, and requirements, respectively. In 1 week, $54.8 \%$ of the foods were substituted; $44.3 \%$ were equivalent quality substitutions, $32.6 \%$ superior, and $23.1 \%$ inferior.

Conclusion: Menus need to provide two thirds of the daily requirement of fiber $(12-16 \mathrm{~g})$ to match the CACFP guidelines. More than $10 \%$ of total calories came from saturated fat and menus did not provide two thirds of total energy requirement (666-1067 kcals). Menus were fairly compliant with the CACFP guidelines however, there is a room for improvement. Foodservice personnel training may enhance nutritional quality of meals and substitutions.

Funding: National Institute on Minority Health and Health Disparities (R01MD011266). 